\title{
Les prélèvements microbiologiques ont-ils encore une place dans le diagnostic de pneumopathie acquise sous ventilation mécanique?
}

\author{
Is there still a place for microbiological sampling for the diagnosis of ventilator-associated \\ pneumonia?
}

\author{
N. Layios $\cdot$ P. Damas \\ Reçu le 18 juillet 2011 ; accepté le $1^{\text {er }}$ novembre 2011 \\ (C) SRLF et Springer-Verlag France 2011
}

Résumé Le diagnostic de la pneumopathie acquise sous ventilation mécanique (PAVM) se base sur un ensemble de signes peu spécifiques. Plusieurs indicateurs ont été testés pour améliorer la performance de ce diagnostic. Aucun ne démontre, à l'heure actuelle, un apport substantiel, que ce soit la quantification des paramètres cliniques par un score tentant de globaliser l'ensemble des informations, le score clinique d'infection pulmonaire (CPIS) ou les marqueurs biologiques recherchés tant dans le sérum comme la procalcitonine ou la protéine $C$ réactive (CRP), que dans le liquide de lavage bronchoalvéolaire (LLBA), comme le soluble Triggering Receptor Expressed on Myeloid Cells 1 (s-TREM 1), l'endotoxine, les fibres d'élastine, la protéine 10 des cellules Clara ou les cytokines pro-inflammatoires. L'examen direct des prélèvements endotrachéaux, et surtout du LLBA, par la mise en évidence de cellules contenant des bactéries apporterait, seul, une information utile. On ne peut donc pas se passer de la microbiologie. Pour citer cette revue : Réanimation 21 (2012).

Mots clés Pneumonie acquise sous ventilation · Biomarqueur - Score clinique d'infection pulmonaire · s-TREM $1 \cdot$ Procalcitonine

\begin{abstract}
Diagnosis of ventilator-associated pneumonia (VAP) is based on non-specific clinical signs. Several indicators have been tested in order to improve the accuracy of VAP diagnosis. The quantification of clinical parameters by using the clinical pulmonary infection score (CPIS), however, failed to improve the specificity of the diagnosis. This was the same for all the biomarkers tested either in the serum (procalcitonin, C-reactive protein) or in the bron-
\end{abstract}

\footnotetext{
N. Layios $(\bowtie) \cdot$ P. Damas $(\bowtie)$

Service de soins intensifs généraux, centre hospitalier universitaire de Liège, université de Liège, domaine universitaire du Sart-Tilman, B-4000 Liège, Belgique e-mail : nathalie.layios@chu.ulg.ac.be,pdamas@chu.ulg.ac.be
}

choalveolar lavage (BAL) fluid [soluble triggering receptor expressed on myeloid cells 1 (sTREM 1), elastin fibers, endotoxin, pro-inflammatory cytokines, Clara cell protein 10]. The microscopic examination of endotracheal samples alone, especially of the BAL fluid, may provide useful information for the detection of infected cells. Thus, microbiology is still needed. To cite this journal: Réanimation 21 (2012).

Keywords Ventilator-associated pneumonia $\cdot$ Biomarker $\cdot$ Clinical pulmonary infection score $\cdot$ sTREM 1 .

Procalcitonin

\section{Introduction}

La pneumopathie acquise sous ventilation mécanique (PAVM) est une infection fréquente, potentiellement grave et qui exige, comme toute infection se développant chez des patients débilités comme peuvent l'être les patients hospitalisés aux soins intensifs, une réponse thérapeutique rapide et appropriée [1]. L'identification des germes responsables de l'infection et l'analyse de leurs sensibilités aux antibiotiques sont des étapes fondamentales pour affirmer le diagnostic et assurer l'adéquation du traitement [2].

On sait cependant que ces étapes demandent du temps et que, le plus souvent, les responsables ne sont pas connus au moment de la prise de décision thérapeutique, puisqu'il faut un délai d'au moins 24 à 48 heures pour l'identification et la détermination des susceptibilités des bactéries. Aussi est-il utile de préciser tout ce qui pourrait permettre, en dehors du diagnostic microbiologique, de confirmer rapidement la présence d'une PAVM suspectée cliniquement. Car le risque existe que, se basant sur des signes cliniques insuffisamment spécifiques, le diagnostic ne soit trop souvent posé conduisant à une surconsommation des antibiotiques [3]. Cette dernière, outre le coût qu'elle implique, a comme inconvénient majeur 
de sélectionner des germes résistants, ce qui hypothèque l'avenir des patients traités, mais aussi celui des autres patients confrontés à un environnement modifié et dangereux.

Nous allons parcourir ce qui dans la littérature a été testé pour améliorer la performance de la stratégie diagnostique des PAVM.

\section{Quantifier les paramètres cliniques}

Le diagnostic de PAVM est évoqué lorsqu'on observe, chez un patient ventilé, l'apparition d'une nouvelle opacité à la radio des poumons en présence d'au moins deux des trois signes suivants : une fièvre, un compte leucocytaire anormalement haut ou bas et un aspect purulent des aspirations endotrachéales. Ce sont les critères de Johanson et al. [4] repris par les sociétés scientifiques et le Center for Diseases Control d'Atlanta [5]. Pugin et al. ont proposé, en 1991, d'ajouter à ces signes l'altération des échanges gazeux estimés par le rapport $\mathrm{PaO}_{2} / \mathrm{FiO}_{2}$ et l'abondance semiquantitative de la culture bactériologique d'un prélèvement distal prélevé à l'aveugle [6]. Attribuant une valeur numérique à chacun de ces six paramètres selon l'importance de leurs perturbations, ils ont créé le score clinique d'infection pulmonaire (CPIS) qui, au-dessus de 6, permet d'affirmer la présence d'une PAVM avec une sensibilité rapportée de $93 \%$ et une spécificité de $100 \%$. Depuis sa publication, ce score est régulièrement cité et utilisé, mais il pose question : sa validation ne rencontre pas l'exigence actuellement attendue pour un score. La pondération des paramètres est arbitraire et ne repose pas sur un nombre important d'observations (28 patients dans l'étude initiale de Pugin et al.). De plus, la modification de l'image radiologique des poumons peut être difficile à juger ; l'évaluation de l'abondance et du caractère purulent des aspirations endotrachéales est subjective et peu reproductible ; l'altération des échanges gazeux repose sur un seuil discutable de $240 \mathrm{mmHg}$ fixé pour le rapport $\mathrm{PaO}_{2} / \mathrm{FiO}_{2}$, qui en outre n'est pas applicable en cas de syndrome de détresse respiratoire aigu (SDRA), situation régulièrement compliquée de surinfection. Il faut de surcroît attendre au moins 24 heures pour évaluer la croissance bactérienne. Enfin, la validation finale du CPIS s'est faite sur la base d'un index bactérien qui est la somme des logarithmes des concentrations des populations bactériennes rencontrées [6]. Cet index n'est plus usité.

Il n'est donc pas étonnant de voir que ceux qui recourent à ce score le modifient tous un tant soit peu, essentiellement en supprimant l'évaluation de la charge bactérienne pour pouvoir l'utiliser dès la suspicion clinique d'infection. Mais la corrélation avec les mesures bactériologiques quantitatives sur le liquide de lavage bronchoalvéolaire (LLBA) est médiocre. Luyt et al. rapportent, dans une étude rétrospec- tive de données collectées pour une étude multicentrique sur le diagnostic des PAVM, que le CPIS n'a qu'une spécificité de $47 \%$ menant à traiter plus de la moitié des patients chez qui une PAVM n'est pas confirmée in fine [7]. La même année, Schurink et al. rapportent une spécificité encore plus basse à $17 \%$ [8]. Ils testent dans le même temps la reproductibilité du score entre observateurs et trouvent une concordance médiocre avec un test Kappa de 0,16. Les auteurs concluent à un usage prudent du CPIS, peu spécifique et peu reproductible. De même, Croce et al. soulignent chez 158 patients traumatisés l'incapacité du CPIS à discriminer les patients souffrant de SIRS (systemic inflammatory response syndrome) de ceux souffrant de PAVM [9]. Miller et al. trouvent, eux, de meilleures performances avec une spécificité de $69 \%$ et une sensibilité de $84 \%$ pour le CPIS dans une population de 292 patients également traumatisés [10].

Le CPIS n'apporte donc pas une réponse adéquate pour améliorer le diagnostic des PAVM. Il repose néanmoins sur l'ensemble des critères que tous les cliniciens évaluent lors de leur travail quotidien au chevet du patient pour rechercher l'éventualité d'une complication infectieuse. Ce sont ces paramètres qui les alertent et qui déclenchent les recherches diagnostiques et les décisions thérapeutiques. On peut penser que l'établissement d'un CPIS systématise une démarche clinique de diagnostic permettant de se forger une conviction plus ou moins forte, ce qui est loin d'être négligeable. Cependant, on peut également espérer qu'un nouveau CPIS soit établi sur un grand nombre d'observations par une étude multicentrique sur des bases statistiques plus solides que celles existantes.

\section{Examen direct des prélèvements}

La qualité d'un prélèvement conditionne les réponses que l'on obtient de lui. L'examen microscopique direct permet de décrire les cellules, d'en évaluer la quantité, d'en définir le type et de rechercher la présence de micro-organismes intracellulaires (polymorphonucléaires neutrophiles [PMN] et macrophages) potentiellement incriminables dans l'infection qu'on investigue. L'aspiration endotrachéale ne doit pas être contaminée par une trop grande quantité de cellules épithéliales qui apportent avec elles la flore colonisatrice de l'oropharynx. Quand des PMN sont par contre observés en quantité, le prélèvement confirme la suspicion clinique d'infection [5]. C'est le LLBA qui a le plus été étudié, car les prélèvements trachéobronchiques sont souvent peu manipulables du fait du mucus, ce qui donne lieu à des interprétations peu reproductibles. Le LLBA permet des colorations qui donnent lieu à des examens beaucoup mieux interprétables et qui ont abouti à proposer comme signe spécifique 
d'infection la présence d'un taux suffisant de cellules contenant des micro-organismes. La littérature, très contrastée, rapportait des proportions de cellules infectées variant de 1 à $15 \%$ à la fin des années 1990 [11] avec adoption par la suite d'un seuil à $2 \%$ selon de nombreuses études [12-14]. Une étude plus récente [15] met en cause, par ailleurs, la validité de ce taux dans les VAP tardives ( $>7$ jours). Ce comptage n'est pas toujours aisé, et les études rapportées ne recourent pas toutes à des références admises. C'est notamment le cas pour la bactériologie quantitative sur le LLBA. Se basant sur les résultats d'une brosse protégée pour définir une PAVM avec un seuil de $10^{3} \mathrm{CFU} / \mathrm{ml}$, Allaouiche et al. rapportent une spécificité diagnostique de $80 \%$ et une sensibilité de $84 \%$ pour le diagnostic de PAVM pour un seuil de $2 \%$ de cellules contenant des micro-organismes [16]. Différentes études reprises dans une revue récente [17] donnent des sensibilités allant de 75 à $86 \%$ et des spécificités allant de 78 à $98 \%$, résultats honorables, même si Vallés et al. remarquent, de façon intéressante en rapportant quatre cas, que cela pourrait ne pas se vérifier dans la PAVM provoquée par Pseudomonas aeruginosa [18]. En effet, ce germe peut être porteur d'une leucocidine destructrice des PMN [19].

\section{Marqueurs biologiques de l'infection}

Une infection pulmonaire implique une réaction de l'hôte contre un ou plusieurs micro-organismes envahissant ses tissus. Cela provoque la mobilisation d'une série de médiateurs endogènes nécessaires à l'élaboration et au contrôle de cette réponse mais aussi la survenue de molécules provenant du dommage tissulaire ou des bactéries infectantes ellesmêmes. Il n'est donc pas étonnant que plusieurs molécules candidates aient été testées pour renforcer la spécificité du diagnostic clinique, que ce soit dans le sang circulant ou au sein du poumon par l'intermédiaire du LLBA.

\section{Marqueurs bactériens}

Recherchant la présence d'endotoxine (lipopolysaccharide [LPS]) dans le LLBA, trois études au moins rapportent que des taux supérieurs à quatre à six unités d'endotoxine par millilitre confirment le diagnostic de PAVM [20-22]. La corrélation avec une bactériologie quantitative n'est assurée que par une seule de ces études [22] avec une excellente sensibilité (82-92\%) et spécificité (83-95\%). Mais cette technique, relativement coûteuse, ne détecte que les PAVM provoquées par les bactéries à Gram négatif, vu la molécule membranaire constitutive. Le peptidoglycane, ubiquitaire, a déjà été détecté dans le sang de patients infectés. Aucune étude n'existe concernant les PAVM.

\section{Nécrose parenchymateuse}

La présence de fibres d'élastine dans le LLBA a été proposée comme témoin de la destruction tissulaire par le processus infectieux [23], mais sa spécificité est faible d'après une autre étude [24].

\section{Marqueurs inflammatoires}

\section{Soluble Triggering Receptor Expressed on Myeloid Cells 1 (s-TREM 1)}

La mesure dans le LLBA de la concentration d'un récepteur soluble exprimée par les cellules myéloïdes (s-TREM 1) a été rapportée par Gibot et al. comme particulièrement discriminante pour le diagnostic de PAVM [25]. Les auteurs ont montré que cette concentration était plus performante que n'importe lequel des signes cliniques ou biologiques usuels, atteignant une sensibilité de $98 \%$ et une spécificité de $90 \%$ chez 148 patients suspectés de souffrir de pneumonie communautaire ou hospitalière. Le diagnostic de PAVM était défini par un seuil de $10^{3} \mathrm{CFU} / \mathrm{ml}$ dans un liquide de miniLBA protégé de $20 \mathrm{ml}$. Le seuil de signification pour le s-TREM était proposé à $5 \mathrm{pg} / \mathrm{ml}$. Cela n'a malheureusement pas été confirmé par Honorenko et al. qui ont trouvé autant de s-TREM 1 dans le LLBA $(5 \times 20 \mathrm{ml})$ de patients avec PAVM que sans PAVM [26], ni par Anand et al. qui ont trouvé en moyenne $171,9 \mathrm{pg} / \mathrm{ml}$ chez les patients atteints de PAVM et $96,7 \mathrm{pg} / \mathrm{ml}$ en absence de PAVM, ce qui n'est pas statistiquement différent [27]. Par ailleurs, Oudhuis et al. ont rapporté des taux moyens aussi élevés que $1424 \mathrm{pg} / \mathrm{ml}$ dans le LLBA de patients sans PAVM confirmée par bactériologie quantitative [28]. On admettra que le seuil est probablement bien plus élevé que celui de $200 \mathrm{pg} / \mathrm{ml}$ proposé par Determan et al. dans un travail où des mini-LBA non dirigés étaient répétés tous les deux jours. La sensibilité de cette procédure était de $75 \%$ et la spécificité de $84 \%$ [29].

\section{Procalcitonine}

La procalcitonine a démontré son utilité pour préciser l'origine bactérienne des pneumopathies communautaires $[30,31]$. Est-ce extrapolable dans le contexte de la PAVM ? Duflo et al. ont montré que, dans le sérum, un taux de procalcitonine supérieur à $3,9 \mathrm{ng} / \mathrm{ml}$ avait une spécificité de $100 \%$, mais une sensibilité de $41 \%$ pour le diagnostic de PAVM par ailleurs basé sur une bactériologie quantitative avec seuil supérieur à $10^{3} \mathrm{CFU} / \mathrm{ml}$ dans un mini-LBA [32]. Il faut noter cependant que le groupe sans PAVM avait un taux moyen égal à $1,5 \mathrm{ng} / \mathrm{ml}$, ce qui, d'après d'autres auteurs, correspond à un critère de traitement antibiotique nécessaire [33]. D'autres observations sont beaucoup plus 
décevantes : ainsi Luyt et al., définissant une PAVM par une bactériologie quantitative avec un seuil supérieur à $10^{4} \mathrm{CFU} / \mathrm{ml}$ dans le LLBA, retrouvé chez 32 patients parmi 73 investigués, ont trouvé une procalcitoninémie plus élevée chez les patients sans PAVM (médiane $1,4 \mathrm{ng} / \mathrm{ml}$ ) que chez les patients avec PAVM (médiane 1,07 pg/ml) [34]. Gibot et al. ont fait une observation similaire dans leur étude sur le s-TREM 1, avec prélèvements sériques pour mesurer la procalcitonémie en parallèle : des concentrations relativement élevées de procalcitonine étaient retrouvées tant chez les patients avec PAVM confirmée $(2,6 \pm 0,8 \mathrm{ng} / \mathrm{ml})$ que chez les patients sans PAVM confirmée $(2,5 \pm 1,2 \mathrm{ng} / \mathrm{ml})$ [25]. C'est aussi le cas de Jung et al. qui se sont récemment posé la question de l'apport de la procalcitonine au diagnostic basé sur un CPIS [35]. Pour ces auteurs, la procalcitonine n'apporte rien, les dosages sont tout à fait superposables entre patients avec PAVM et sans PAVM.

Il faut rappeler que la littérature récente tend à prouver un apport plus substantiel de ce marqueur biologique en termes de durée de traitement antibiotique nécessaire plutôt qu'en termes d'outil de stratégie diagnostique [36].

\section{La protéine $\mathrm{C}$ réactive (CRP)}

La CRP est la protéine de la phase aiguë la plus mesurée pour évaluer la présence et déterminer l'évolution d'une réaction inflammatoire [37]. Elle ne peut prétendre discriminer une origine infectieuse d'une origine non infectieuse, ni prédire la gravité de la pathologie [38]. Il n'en reste pas moins vrai qu'on s'attend à ce qu'elle soit élevée lors du diagnostic de PAVM, et c'est son absence de modification récente qui devrait être testée pour rejeter le diagnostic de PAVM. C'est une piste d'investigation future possible.

\section{Autres marqueurs}

Les cytokines pro-inflammatoires sont par nature des candidates intéressantes pour la stratégie diagnostique des PAVM. L'interleukine (IL)-1béta et l'IL-8 ont été mesurées avec d'autres dans le LLBA de patients suspects de PAVM par Conway Morris et al. [39]. Ils ont montré qu'un taux inférieur à $10 \mathrm{pg} / \mathrm{ml}$ pour l'IL-1béta était bien corrélé à l'absence de PAVM, alors qu'un taux supérieur à $2 \mathrm{ng} / \mathrm{ml}$ pour l'IL-8 était bien corrélé à la présence de PAVM. Curieusement, la combinaison des deux mesures n'améliorait pas le pouvoir de discrimination apporté par une seule mesure prise isolément. Cette observation demande à être confirmée selon les auteurs eux-mêmes, d'autant plus qu'une partie des PAVM reconnues étaient provoquées par des Candida species ( $15 \%$ des cas), ce qui est une entité tout à fait discutable et discutée [40].

La copeptine [41] et un fragment de la molécule mère du facteur natriurétique atrial [42] ont également été testés, mais pour définir la gravité de la PAVM plutôt que pour asseoir son diagnostic. La protéine 10 des cellules Clara a été également testée dans le LLBA, mais n'apparaît pas discriminante [43]. L'inhibiteur de l'activateur du plasminogène, le PAI1, vient de faire l'objet d'une étude chez 32 enfants en association avec le s-TREM 1, les receptor for advanced glycation end-products (RAGE) et une protéine du surfactant, la protéine D. L'inhibiteur de l'activateur du plasminogène 1 (PAI1) montrerait le meilleur pouvoir discriminant pour la présence de micro-organismes dans les prélèvements [44].

Une dernière observation intéressante, mais qui nécessite confirmation, est le suivi de la production de monoxyde d'azote (NO) par la muqueuse nasale qui permettrait de discriminer les patients développant une PAVM [45].

\section{Commentaires}

Un des problèmes posés par la PAVM reste le diagnostic. Les études les plus crédibles se basent sur des mesures bactériologiques quantitatives qui ne font pas partie de la batterie de tests utilisés en routine clinique aux soins intensifs [46] et qui sont par ailleurs toujours controversées [47]. Il apparait, après cette revue des indicateurs proposés pour améliorer le diagnostic des PAVM, qu'aucun ne se révèle suffisamment robuste $[48,49]$. Seuls l'examen direct négatif et l'absence de mise en évidence de germes intracellulaires semblent se révéler utiles pour proposer de repousser une antibiothérapie chez les patients dont l'état reste stable. Les critères cliniques sont ceux sur lesquels le clinicien se base pour démarrer sa réflexion. Il ne devrait pas s'en contenter, mais peu de choses s'offrent à lui pour étayer sa démarche. Assurer un prélèvement adéquat avant toute antibiothérapie ou toute modification de celle-ci relève d'une bonne pratique clinique, et cette démarche est facilitée par le fait que les patients sont intubés. Le liquide de LBA permet à la fois un examen direct de qualité, une mesure bactériologique quantitative et une analyse cytologique pouvant déboucher sur d'autres diagnostics. Cet examen, le plus riche d'enseignement, devrait être privilégié. Classiquement, on le réserve aux cas difficiles ou aux immunodéprimés, on devrait à notre sens y recourir plus volontiers pour tous les cas ne nécessitant pas un traitement empirique immédiat comme l'exigent les états de sepsis sévère ou les chocs septiques [50].

\section{Conclusion}

À l'heure actuelle, il n'existe pas d'indicateurs spécifiques permettant de corroborer le diagnostic clinique de la PAVM. Le diagnostic clinique se base sur les critères du CPIS qui, tel qu'il est formulé, ne permet pas non plus d'être plus 
assuré. L'examen direct d'un bon prélèvement par la recherche des bactéries intraleucocytaires apporterait une information utile. On ne peut se passer de la microbiologie pour confirmer le diagnostic et assurer l'adéquation du traitement.

Conflit d'intérêt : les auteurs déclarent ne pas avoir de conflit d'intérêt.

\section{Références}

1. Klompas M (2007) Does this patient have ventilator-associated pneumonia? JAMA 297:1583-93

2. Chastre J, Fagon J (2002) Ventilator associated pneumonia. Am J Resp Crit Care Med 165:867-903

3. Chastre J, Trouillet JL, Combes A, Luyt CE (2010) Diagnostics techniques and procedures for establishing the microbial etiology of ventilator-associated pneumonia for clinical trials: the pros for quantitative cultures. Clin Infect Dis 51:S88-S92

4. Johanson WG Jr, Pierce AK, Sanford JP, Thomas GD (1972) Nosocomial respiratory infections with gram-negative bacilli. The significance of colonization of the respiratory tract. Ann Intern Med 77:701-6

5. 2005) Guidelines for the management of adults with hospital acquired, ventilator-associated, and healthcare-associated pneumonia. Am J Respir Crit Care Med 171:388-416

6. Pugin J, Auckenthaler R, Mili N, et al (1991) Diagnosis of ventilator-associated pneumonia by bacteriologic analysis of bronchoscopic and non bronchoscopic "blind" bronchoalveolar lavage fluid. Am Rev Respir Dis 143:1121-9

7. Luyt CE, Chastre J, Fagon JY (2004) Value of the clinical pulmonary infection score for the identification and management of ventilator-associated pneumonia. Intensive Care Med 30:844-52

8. Schurink CA, Van Nieuwenhoven CA, Jacobs JA, et al (2004) Clinical pulmonary infection score for ventilator-associated pneumonia: accuracy and inter-observer variability. Intensive Care Med 30:217-24

9. Croce MA, Swanson JM, Magnotti LJ, et al (2006) The futility of the clinical pulmonary infection score in trauma patients. J Trauma 60:523-7

10. Miller PR, Meredith JW, Chang MC (2003) Optimal threshold for diagnosis of ventilator associated pneumonia using bronchoalveolar lavege. J trauma 55:263-7

11. De Jaeger A, Litalien C, Lacroix J, et al (1999) Protected specimen brush or bronchoalveolar lavage to diagnose bacterial nosocomial pneumonia in ventilated adults: a meta-analysis. Crit Care Med 27:2548-60

12. Combes A, Figliolini C, Trouillet JL, et al (2002) Incidence and outcome of polymicrobial ventilator-associated pneumonia. Chest 121:1618-23

13. Hayon J, Figliolini C, Combes A, et al (2002) Role of serial routine microbiologic culture results in the initial management of ventilator-associated pneumonia. Am J Respir Crit Care Med $165: 41-6$

14. Trouillet JL, Vuagnat A, Combes A, et al (2002) Pseudomonas aeruginosa ventilator-associated: comparison of episodes due to piperacillin-resistant versus piperacillin-susceptible organisms. Clin Infect Dis 34:1047-54

15. Dupont H, Chalhoub V, Plantefève G, et al (2004) Variation of infected cell count in bronchoalveolar lavage and timing of ventilator-associated pneumonia. Intensive Care Med 30:1557-63

16. Allaouchiche B, Jaumain H, Dumontet C, Motin J (1996) Early diagnosis of ventilator-associated pneumonia. Is it possible to define a cutoff value of infected cells in BAL fluid? Chest 110: $1558-65$

17. Rea-Neto A, Youssef NC, Tuche F, et al (2008) Diagnosis of ventilator-associated pneumonia: a systematic review of the literature. Crit Care 12:R56

18. Vallés J, Rello J, Fernandez R, et al (1994) Role of bronchoalveolar lavage in mechanically ventilated patients with suspected pneumonia. Eur J Microbiol Infect Dis 13:549-58

19. LeBerre R, Nguyen S, Nowak E, et al (2011) Relative contribution of three main virulence factors in pseudomonas aeruginosa pneumonia. Crit Care Med. Epub ahead of print

20. Pugin J, Auckenthaler R, Delaspre O, et al (1992) Rapid diagnosis of Gram negative pneumonia by assay of endotoxin in bronchoalveolar lavage fluid. Thorax 47:547-9

21. Flanagan PG, Jackson SK, Findlay G (2001) Diagnosis of gram negative, ventilator associated pneumonia by assaying endotoxin in bronchial lavage fluid. J Clin Pathol 54:107-10

22. Nys M, Ledoux D, Canivet JL, et al (2000) Correlation between endotoxin level and bacterial count in bronchoalveolar lavage fluid of ventilated patients. Crit Care Med 28:2825-30

23. el-Ebiary M, Torres A, Gonzalez J, et al (1995) Use of elastin fibre detection in the diagnosis of ventilator-associated pneumonia. Thorax 50:14-7

24. Shepherd KE, Lynch KE, Wain JC, et al (1995) Elastin fibers and the diagnosis of bacterial pneumonia in the adult respiratory distress syndrome. Crit Care Med 23:1829-34

25. Gibot S, Cravoisy A, Levy B, et al (2004) Soluble triggering receptor expressed on myeloid cells and the diagnosis of pneumonia. N Engl J Med 350:451-8

26. Horonenko G, Hoyt J, Robbins R, et al (2007) Soluble triggering receptor expressed on myeloid cell-1 is increased in patients with ventilator-associated pneumonia: a preliminary report. Chest 132:58-63

27. Anand N, Zuick S, Klesney-Tait J, Kollef M (2009) Diagnostic implications of soluble triggering receptor expressed on myeloid cells-1 in BAL fluid of patients with pulmonary infiltrates in the ICU. Chest 135:641-7

28. Oudhuis G, Beuving J, Bergmans D, et al (2009) Soluble triggering receptor expressed on myeloid cells-1 in bronchoalveolar lavage fluid is not predictive for ventilator-associated pneumonia. Intensive Care Med 35:1265-70

29. Determann RM, Millo JL, Gibot S, et al (2005) Serial changes in soluble triggering receptor expressed on myeloid cells in the lung during development of ventilator-associated pneumonia. Intensive Care Med 31:1495-500

30. Christ-Crain M, Stolz D, Bingisser R, et al (2006) Procalcitonin Guidance of Antibiotic Therapy in Community-acquired Pneumonia A Randomized Trial. Am J Respir Crit Care Med 174:84-93

31. Schuetz P, Christ-Crain M, Thomann R, et al (2009) Effect of procalcitonin-based guidelines vs standard guidelines on antibiotic use in lower respiratory tract infections: the ProHOSP randomized controlled trial. JAMA 302:1059-66

32. Duflo F, Debon R, Monneret G, et al (2002) Alveolar and serum procalcitonin: diagnostic and prognostic value in ventilatorassociated pneumonia. Anesthesiology 96:74-9

33. Schuetz P, Christ-Crain M, Muller B (2007) Biomarkers to improve diagnostic and prognostic accuracy in systemic infections. Curr Opin Crit Care 13:578-85

34. Luyt CE, Combes A, Reynaud C, et al (2008) Usefulness of procalcitonin for the diagnosis of ventilator-associated pneumonia. Intensive Care Med 34:1434-40

35. Jung B, Embriaco N, Roux F, et al (2010) Microbiogical data, but not procalcitonin improve the accuracy of the clinical pulmonary infection score. Intensive Care Med 36:790-8 
36. Bouadma L, Luyt CE, Tubach F, et al (2010) Use of procalcitonin to reduce patients' exposure to antibiotics in intensive care unit (PRORATA trial): a multicentre randomized, controlled trial. Lancet 375:463-74

37. Póvoa P, Coelho L, Almeida E, et al (2005) C reactive protein as a marker of ventilator-associated pneumonia resolution: a pilot study. Eur Respir J 25:804-12

38. Oppert M, Reinicke A, Muller C, et al (2002) Elevations in procalcitonin but not Creactive protein are associated with pneumonia after cardiopulmonary resuscitation. Resuscitation 53:167-70

39. Conway Morris A, Kefala K, Wilkinson TS, et al (2010) Diagnostic importance of pulmonary interleukin- $1 \mathrm{~b}$ and interleukin-8 in ventilator-associated pneumonia. Thorax 65:201-7

40. Ricard JD, Roux D (2009) Candida pneumonia in the ICU: Myth or reality? Intensive Care Med 35:1500-12

41. Seligman R, Papassotiriou J, Morgenthaler NG, et al (2008) Copeptin, a novel prognostic biomarker in ventilator-associated Pneumonia. Critical Care 12:R11

42. Seligman R, Papassotiriou J, Nils G, et al (2008) Prognostic value of midregional pro-atrial natriuretic peptide in ventilatorassociated pneumonia. Intensive Care Med 34:2084-91

43. Vanspauwen MJ, Linssen CF, Bruggeman CA, et al (2011) Clara cell protein in bronchoalveolar lavage fluid: a predictor of ventilator-associated pneumonia? Critical Care 15:R14
44. Srinivasan R, Song Y, Wiener-Kronish J, et al (2011) Plasminogen activation inhibitor concentrations in bronchoalveolar lavage fluid distinguishes ventilator-associated pneumonia from colonization in mechanically ventilated pediatric patients. Pediatr crit care med 12:21-7

45. Tadié JM, Trinquart L, Jannière-Nartey C, et al (2010) Prediction of nosocomial infection acquisition in ventilated patients by nasal nitric oxide: proof-of-concept study. Shock 34:217-21

46. Koulenti D, Lisboa T, Brun-Buisson, et al (2009) Spectrum of practice in the diagnosis of nosocomial pneumonia in patients requiring mechanical ventilation in European intensive care unit. Crit Care Med 37:2360-8

47. Niederman MS (2010) The Argument against Using Quantitative Cultures in Clinical Trials and for the Management of VentilatorAssociated Pneumonia. Clin Infect Dis 51:S93-S9

48. Shorr A, Chan CM, Zilberberg MD (2011) Diagnostics and epidemiology in ventilator-associated pneumonia. Ther Adv Respir Dis 5:121-30

49. Palazzo SJ, Simpson T, Schnapp L (2011) Biomarkers for ventilator-associated pneumonia: Review of the literature. Heart and Lung 40:293-8

50. Damas P, Layios N, Seidel L, et al (2011) Severity of ICUacquired pneumonia according to infectious microorganisms. Intensive Care Med 37:1128-35 